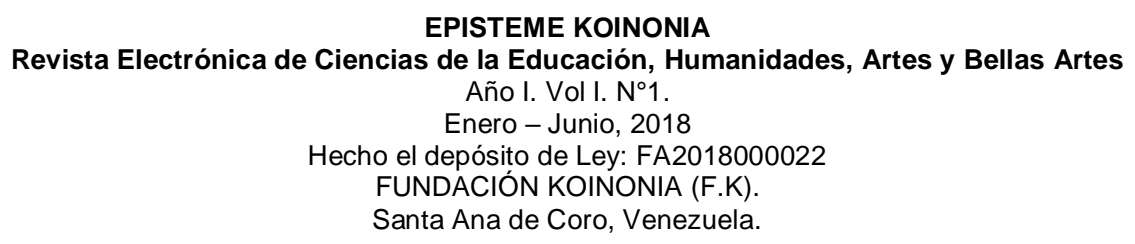

Alvis José Garcia Morales; Caroll Lisette Gonzalez Ten Meer

\title{
La investigación acción participativa (IAP), como herramienta para identificar la gestión de la calidad docente
}

\section{Participatory action research (IAP), as a tool to identify management of teaching quality}

\author{
Alvis José Garcia Morales \\ alvis7114@gmail.com \\ Universidad Valle de Momboy, Valera \\ Venezuela \\ Caroll Lisette Gonzalez Ten Meer \\ greg7114@gmail.com \\ Universidad Valle de Momboy, Valera \\ Venezuela
}

Recibido: 12 de octubre de 2019

Aprobado: 15 de septiembre de 2017

\begin{abstract}
RESUMEN
El objetivo del presente artículo consiste en el estudio de la Gestión de la calidad académica docente mediante la Investigación Acción Participativa (IAP), basándose en el análisis crítico y documental del pensamiento referido a los autores, González (2013), Hurtado (2012), McKernan (2001), Dos santos y Sánchez (2001) a través de los cuales reflexionan sobre la necesidad de impulsar la IAP considerando el ámbito educativo como la base fundamental para un aprendizaje dialógico e interpretativo de la gestión del docente, y poder tomar conciencia de la calidad académica que se dicta dentro de los diferentes ambientes de aprendizaje. Para lograr dicha aplicación se debe destacar que el principio más importante de la teoría del conocimiento es el tratar los hechos sociales como cosas, como objetos del conocimiento. Los datos basados en la observación y en experimentos son necesarios para su comprensión Durkheim (1982), por lo que se resalta para alcanzar la humanización y la transformación de los saberes, ser auténticos y revestidos de acción, amor, humildad, por lo que se acentúa que el individuo cuenta con capacidades propias de un ser pensante y autocritico para cambiar la realidad. Se finaliza reflexionando que nuevos tiempos implican nuevas exigencias, por lo cual la gestión del docente para una calidad académica no se está dando, ya que muchos en sus ambientes continúan con la misma rutina diaria. Su desempeño se basa en estrategias e instrumentos que hacen de su desempeño una monotonía, ocasionando una reducción a descubrir el beneficio de aprender a pensar.
\end{abstract}

Descriptores: Investigación acción participativa (IAP); Herramienta; Gestión; Calidad docente; Docente. 


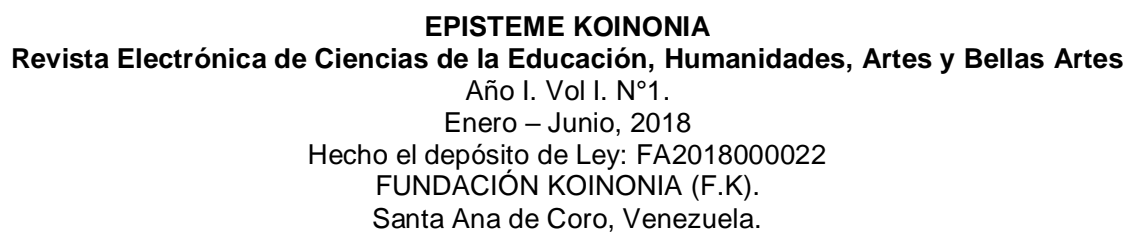

Alvis José Garcia Morales; Caroll Lisette Gonzalez Ten Meer

\begin{abstract}
The objective of this article is to study the management of academic quality through the Participatory Action Research (IAP), based on the critical and documental analysis of the thinking referred to the authors, González (2013), Hurtado (2012), McKernan (2001), Dos Santos and Sanchez (2001), through which they reflect on the need to promote the IAP considering the educational field as the fundamental basis for a dialogic and interpretative learning of teacher management, and to become aware of the academic quality that is dictated within the different environments of learning. To achieve this application it should be noted that the most important principle of the theory of knowledge is to treat social facts as things, as objects of knowledge. The data based on observation and experiments are necessary for Durkheim (1982) understanding, so it is emphasized to reach humanization and the transformation of knowledge, to be authentic and clothed with action, love, humility, so that Emphasizes that the individual has the capacity of a thinking and self-critical being to change reality. It ends by reflecting that new times imply new demands, so that the management of the teacher for an academic quality is not taking place, since many in their environments continue with the same daily routine. Their performance is based on strategies and instruments that make their performance a monotony, causing a reduction to discover the benefit of learning to think.
\end{abstract}

Descriptors: Participatory action research (IAP); Tool; Management; Teaching quality; Teacher.

\title{
INTRODUCCIÓN
}

Uno de los aspectos más importante dentro de todo proceso educacional enmarcado en una calidad educativa, debe estar basado en una gestión de calidad académica donde se planifique, ejecute y sistematice una serie de pasos que conlleven al logro de los objetivos establecidos por las diferentes instituciones del país. Así pues, la necesidad de que exista una gestión es para mejorar el desempeño laboral docente, en donde deben establecerse cambios significativos en ese producto que sale de las diferentes instituciones educativas. Lo que genera un reto que muchos hoy en día ven que se está debilitando en sus raíces y valores, lo que amerita la pronta intervención de personas que dinamicen ese potencial educativo por parte de los diferentes docentes, destacando su labor. Por esta razón se hace mención Genesi y Suarez (2009: 4), quienes destacan; 


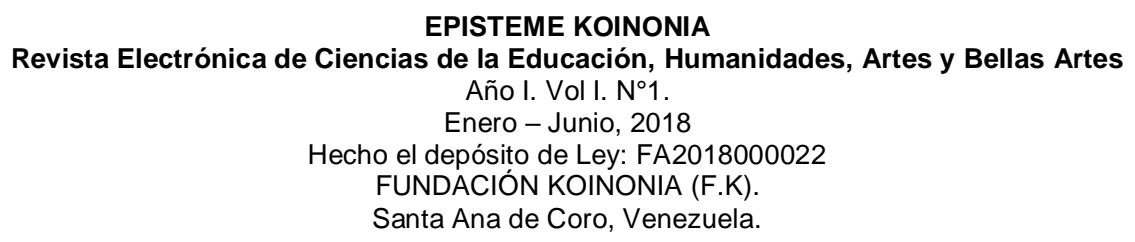

Alvis José Garcia Morales; Caroll Lisette Gonzalez Ten Meer

Las características de la nueva organización imponen nuevas demandas y generan desafíos al sistema educativo. Es por eso, que debe existir correspondencia entre la orientación educativa ofrecida por el sistema nacional y las exigencias de la sociedad actual para promover la creatividad, innovación y experimentación con procesos dirigidos a aumentar la gestión de calidad del sistema educativo.

Dentro de esta misma perspectiva, la presente investigación se ha propuesto conocer el verdadero desempeño laboral del docente como gerente mediante una gestión de calidad académica. No obstante, es necesario una indudable identificación de cada uno de estos como educadores de la generación de relevo por medio de un trabajo innovador, creativo, motivacional, que haga de su participación un acercamiento al cambio que mediante su gestión se pueda lograr, lo que implica el aporte de nuevas ideas, pensamientos, dinamismo, lo cual son parte necesaria dentro y fuera de las instituciones educativas.

Cabe destacar, que es necesario que el docente cumpla con un desempeño laboral considerando las necesidades en las diferentes instituciones, ameritando así, de la aplicación de acciones basadas en su formación como profesional de la docencia, y que cada día la sociedad exige mediante una conducción que considere necesario contextualizar bajo un enfoque sistémico, dinámico, proactivo y con una diversidad de estrategias que lo motiven a asumir un reto dentro de la gestión de la calidad académica.

Es así, que se hace necesaria la aplicación de la Investigación Acción Participativa como principal herramienta para visualizar desde una perspectiva integral la problemática existente y poder concretar los objetivos planteados, generando un interese en el que se involucre el colectivo docente de una forma directa. Así pues, la IAP se toma como parte esencial para estudiar la realidad humana, ligando de esta manera el enfoque experimental e la ciencia social con programas de acción, es por esto que se tiene a González (2013: 17), "Es una forma de investigación que permite vincular el estudio de los problemas en un contexto determinado con programas de 


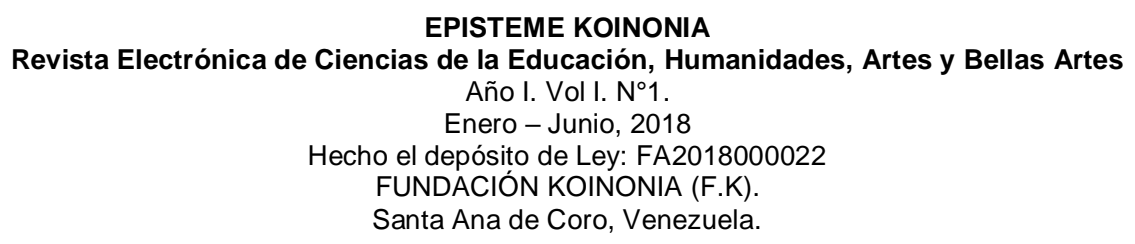

Alvis José Garcia Morales; Caroll Lisette Gonzalez Ten Meer

acción social, de manera que se logren de forma simultánea conocimientos y cambios sociales".

Del mismo modo, se importante la indagación introspectiva de los protagonistas de tal situación social, con el objeto de mejorar las practicas ejecutadas hasta el momento y así transformar el entorno social y educativo del docente, González (2013: 18), "La IAP es definida como una forma de indagación introspectiva colectiva emprendida por participantes en situaciones sociales con objeto de mejorar la racionalidad y la justicia de sus prácticas sociales y de las situaciones en que estas tienen lugar". Al describir lo expuesto por el autor, la necesidad de una participación permite obtener un conocimiento en colectivo de una realidad, conllevando todo esto a un estudio en el que se pueda contar con la participación de todos y cada uno, Rodríguez y otros, (citado por González 2013: 20), "La investigación acción participativa se caracteriza por un conjunto de principios, normas y procedimientos metodológicos que permiten obtener conocimientos colectivos sobre una determinada realidad social".

En este sentido, el progreso dentro del proceso de enseñanza y aprendizaje, debe dar realce a la gestión de la calidad académica, lo que podrá generar una articulación con el desempeño laboral ejercida por los docentes, en donde se puedan plantear nuevas estrategias que dinamicen la innovación de un nuevo aprendizaje significativo en los estudiantes. De acuerdo a esto Hurtado (2012: 125) describe; "La investigación acción es aquella cuyo objetivo consiste en modificar el evento estudiado, generando y aplicando sobre él una intervención especialmente diseñada", es decir, sustituir un estado de una realidad por otra que lleve a mejorar la problemática existente, fundamentada y orientada a la participación de la comunidad estudiada.

Bajo esta misma perspectiva, se considera una de las características dentro de la gestión de calidad académica, en donde se debe considerar el pensamiento crítico y el perfil de la persona que va a gestionar un aprendizaje en los estudiantes de una manera significativa, lo cual es considerado como primordial para una cambio de pensamiento desde la gerencia, Hurtado (2012: 127),..."se inicia con los estadios 


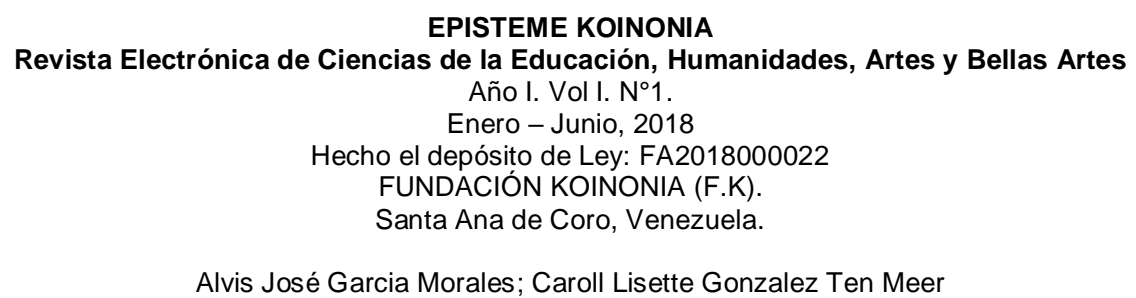

exploratorios y descriptivo, pero no se limita a eso, intenta además proponer y cambiar. Incorpora lo explicativo en la medida en que puede identificar los cambios casados por la acción emprendida y diferenciarlos de los cambios producidos por otros factores". Esta medida de "identificar" es una propuesta para lograr los cambios que se desean realizar en el presente considerando la verdadera labor dentro de la gestión académica que cada docente está realizando dentro de los diferentes ambientes de aprendizaje. Como se puede destacar, la investigación participativa ha intentado hacer comprender una situación que se esté presentando en el tiempo e investigarla, lo que genera la necesidad de mejorarla para una calidad de vida adecuada en los individuos, utilizando los diferentes entornos de la sociedad, como los son empresariales, industriales, educativos y patrones de conductas que cada día se desmoronan afectivamente. McKernan (2001: 23);

La investigación acción es una práctica en la que no se hacen distinciones entre la práctica que se está investigado y el proceso de investigarla. Es decir, enseñar e investigar en la enseñanza no constituyen dos enseñanzas distintas. El propósito último de la investigación es comprender; y comprender es la base de la acción para la mejora".

Lo descrito anteriormente, hace pensar en una estructura investigativa mediante la aplicación de la IAP como herramienta de solución ante cualquier problema que como investigadores se desee dar solución, haciendo de esta la principal fuente de información ante un modelo que busca explicar la acción humana. De la misma forma, se observa el modelo establecido por Ebbut en relación a la Investigación Acción, en donde plantea que la mejor manera de pensar en el proceso es hacerlo. 


\section{EPISTEME KOINONIA}

Revista Electrónica de Ciencias de la Educación, Humanidades, Artes y Bellas Artes

Año I. Vol I. N¹.

Hecho el depósito de Ley: FA2018000022

FUNDACIÓN KOINONIA (F.K)

Santa Ana de Coro, Venezuela.

Alvis José Garcia Morales; Caroll Lisette Gonzalez Ten Meer

Modelo de investigación-acción de Ebbut.

Cuadro 1.

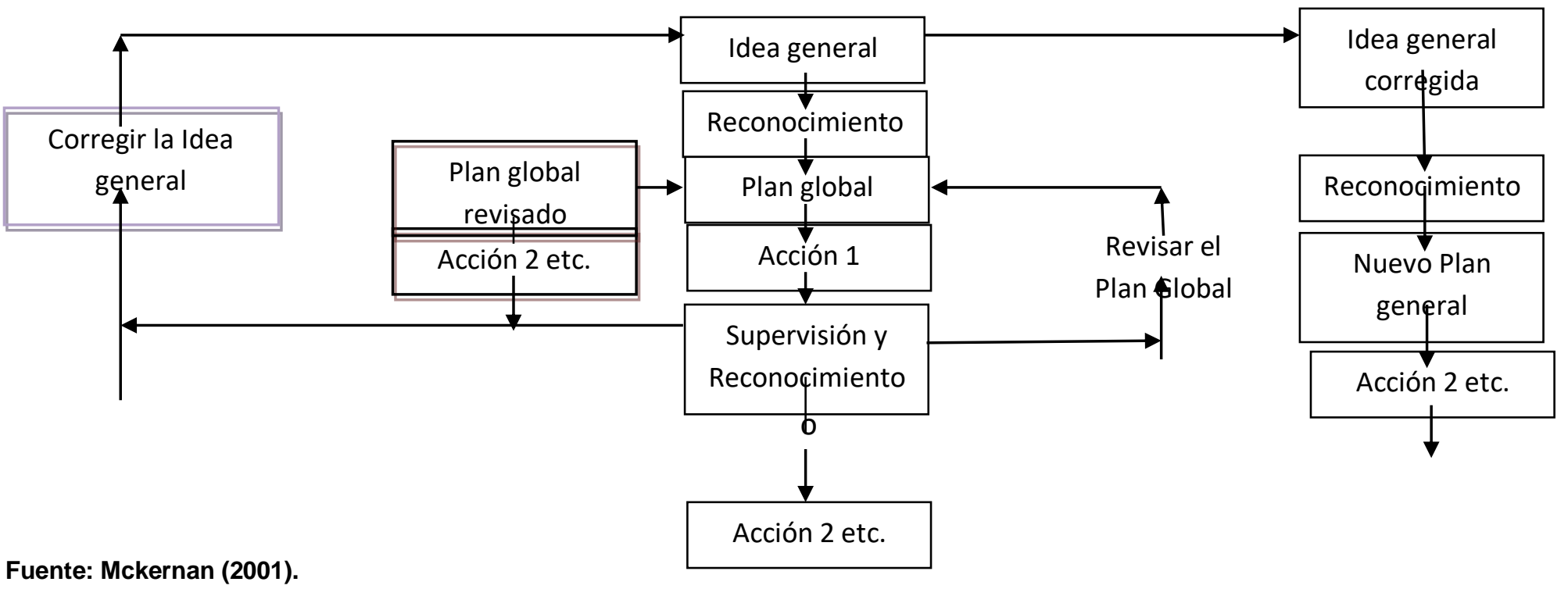




\section{Revista Electrónica de Ciencias de la Educación, Humanidades, Artes y Bellas Artes}

Año I. Vol I. No1.

Hecho el depósito de Ley: FA2018000022

FUNDACIÓN KOINONIA (F.K)

Santa Ana de Coro, Venezuela.

Alvis José Garcia Morales; Caroll Lisette Gonzalez Ten Meer

Modelo de Investigación Acción de McKernan.

\section{Cuadro 2.}

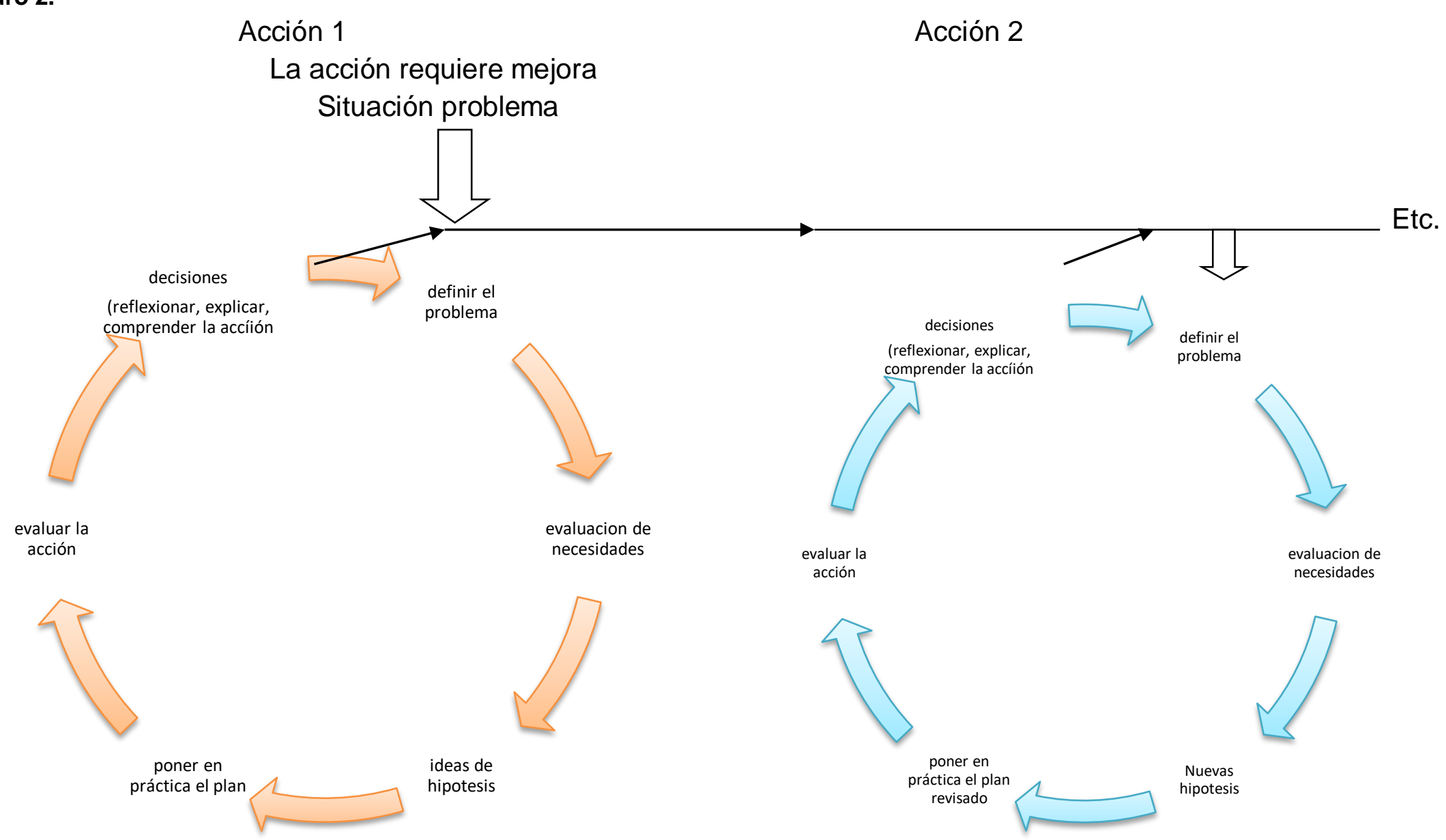

Fuente: McKernan (1988)

Desarrollar el plan de acción

Revisar el plan revisado 


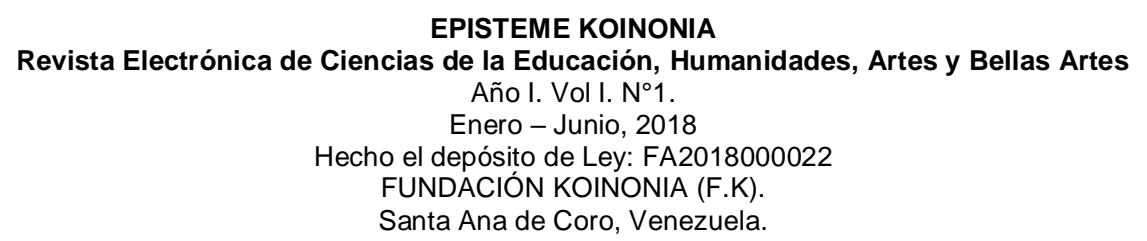

Alvis José Garcia Morales; Caroll Lisette Gonzalez Ten Meer

Dentro del mismo contexto investigativo, se debe considerar, que la educación constituye una de las partes más importantes para lograr la firmeza y la expansión de un proceso educativo de calidad dentro de la sociedad, lo cual, es un desafío necesario en la conformación de una enseñanza que permita mediante la organización y una gestión de calidad, adaptarse a los nuevos cambios que se están dando, por lo que la organización dentro de un desempeño laboral docente, puede considerarse de gran relevancia. Para Durkheim, (citado por Dos santos y Sánchez 2001) "La habilidad para correlacionar hechos sociales es la meta de la ciencia social porque esto permite descubrir las causas tornando las ciencias sociales semejantes a las ciencias de la naturaleza.

Al respecto se considera que el carácter de los hechos sociales no puede ser visto por el investigador con un sentido común, sino que debe colocarse dentro de la problemática existente, para vivenciar cada día lo que realmente está sucediendo, así lo argumenta Dos santos y Sánchez (2001: 20);

... el científico social conoce los hechos sociales no a través del sentido común, que puede variar mucho dentro de determinada sociedad, sino a través de un lenguaje propio separado del lenguaje común y a través de un método propio diferente de los abordajes comunes de conocimiento

Mostradas estas consideraciones, se hace del saber la necesidad de formular nuevas políticas educativas, que integren y orienten el desarrollo personal y profesional de cada docente, para mejorar, actualizar y sostener la calidad académica mediante una labor docente. Es por esto, que es fundamental considerar la condición en la que se encuentra la gestión a nivel educativo en nuestros días, lo que establece que se deben dar cambios prósperos y de gran envergadura para poder mostrar al mundo la calidad que aquí se imparte en cada ambiente de aprendizaje.

\section{METODOLOGÍA}

Para la realización del presente artículo se procedió a utilizar la metodología del análisis crítico basado en la técnica del análisis documental; en el que se resalta el pensamiento 


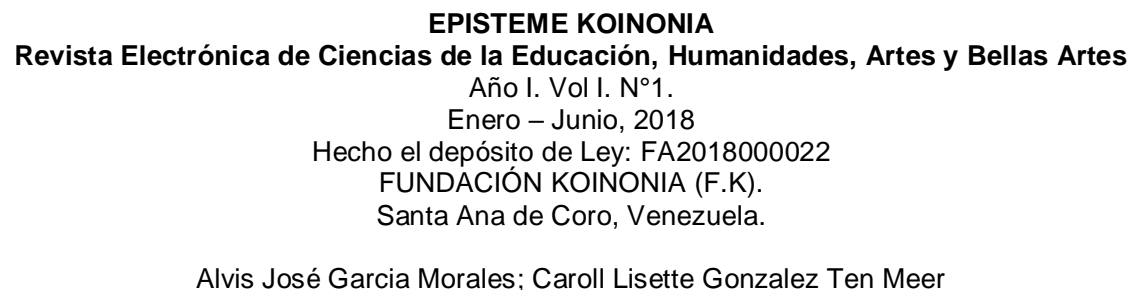

de los siguientes autores González (2013), Hurtado (2012), McKernan (2001), Dos santos y Sánchez (2001) en lo que se refiere a la necesidad de impulsar la IAP para gestionar la calidad académica en los docentes, lo cual en nuestros días es necesario reforzar mediante la IAP, la cual es una gran herramienta para visualizar desde una perspectiva más integral la verdadera problemática existente. Así mismo, se efectúo una revisión de literatura que sirvieron de apoyo relacionados con el estudio que fueron fichadas como principales aportes.

En este mismo aspecto, los resultaos obtenidos más relevantes están relacionados con entender que la única forma para que la educación se convierta en un elemento de gran importancia es dando a los docentes las herramientas que pueda utilizar para una educación que genere un futuro próspero para el estudiante, lo cual implica un índice académico sustentable, lo que implica que el educando pase a ser libre de pensar, analizar, interpretar, reflexionar y transformar su propio proceso de aprendizaje, lo cual instaura al docente a que asuma un pensamiento autentico a través del cual fluyan nuevas ideas e innovaciones para enseñar una esperanza posible de cambiar y mejorar la calidad educativa en cada uno de ellos y ellas.

\section{Gestión}

En el marco de la gestión educativa, se tomaron varios autores quienes la describen dando su opinión en lo que respecta, a como se debe evidenciar y gestionar una administración considerando una serie de estrategias que se pueden utilizar, lo cual debe contribuir a la gestión de calidad académica. En tal sentido se tiene a Alfiz (2006: 142),

La gestión es la verdadera conducción de los proyectos. El hacer se vincula con el pensar, rediseñar y evaluar en un continuo. Hablar de gestión es ver la tarea de la organización escolar desde su dialéctica: implica una ruptura con la lógica de los momentos de la planificación normativa, con la lógica de la administración tradicional (planificación-ejecución-evaluación). 


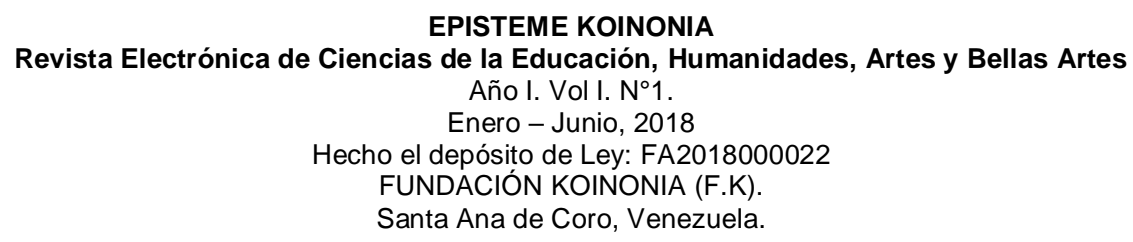

Alvis José Garcia Morales; Caroll Lisette Gonzalez Ten Meer

Como se ha descrito, es tomar en consideración la gestión mediante un cambio de paradigma educativo, en donde el desempeño laboral docente juegue un papel importante para el logro de los objetivos planteados, lo cual implica una praxis docente de calidad, generando un equilibrio para el aprendizaje de los diferentes actores del proceso educativo. Así pues, se debe concretar toda una gestión de calidad académica que ayude a que cada desempeño por parte del docente se vea cristalizado en un corto tiempo.

Bajo este mismo aspecto se destaca la importancia de una gestión global considerando la calidad académica que debe implicar un vínculo entre las dimensiones y su interdependencia de cada uno de los actores educativos, así pues se tiene a Alfiz (2006: 143), el cual describe:

La gestión escolar es la gestión del proyecto global, es decir que implica todas las dimensiones y su interdependencia, así como todos sus actores y sus vínculos. Debe caminar en busca de una coherencia cada vez mayor entre los diferentes aspectos de la vida de la organización y a la vez buscando acortar la distancia entre los enunciados propuestos en la planificación y las acciones reales que tienen lugar en la escuela.

En el marco de lo ya descrito, la gestión de calidad se debe iniciar desde los espacios de aprendizaje, lo que antes era mencionado como aulas, ya que es allí donde el docente aprende a delegar funciones y a establecer acuerdos con sus estudiantes, lo cual amerita de una verdadera gestión escolar.

\section{Gestión de Calidad académica}

Dentro de la cultura escolar que hoy nace se destaca una visión capaz de conceptualizar el proceso educativo mediante una gestión de calidad que pretende que cada docente tenga dinamismo para mejorar la calidad académica de cada estudiante dentro de las diferentes instituciones educativas, de manera que se dé respuesta inmediata a los objetivos que se plantean. Además, la nueva reforma educativa invita a que cada docente tenga una preparación y concientización de su desempeño requerida para un cambio educativo. En este sentido se tiene a Genesi y Suarez (2010: 117); 


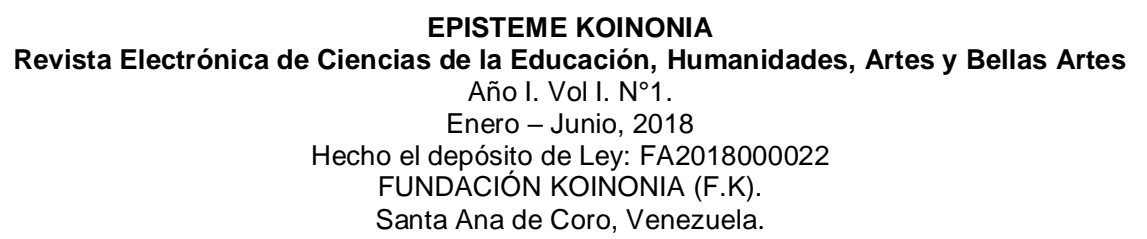

Alvis José Garcia Morales; Caroll Lisette Gonzalez Ten Meer

Por ello, el proceso de formación de profesionales docentes exige de una conducción que considere como requisito esencial su carácter contextualizado, un enfoque sistémico y dinámico. Esta realidad impone un reto en la gestión docente donde se tienen que asumir estrategias didácticas alejadas de los estilos de dirección tradicionales, y pueda abocarse a la función orientadora como criterio formativo...

Considerando lo expuesto, se debe pronunciar una cultura escolar formada por nuevos enfoques sistémicos y dinámicos que generen un cambio significativo dentro del sistema educativo, es por eso que es necesario dar inicio a cambios desde las raíces, lo que genera la atención de todos y cada uno de los docentes asumiendo de esta manera nuevas estrategias de enseñanza y aprendizaje y de este modo lograr una gestión de calidad académica.

\section{Desempeño laboral docente}

Bajo este mismo pensamiento, se describe el verdadero rol del docente desde su desempeño laboral o praxis educativa; por lo que muchos son los pensamientos que hacen precisar la varadera misión del docente, en la cual se debe destacar las actitudes y habilidades en el diseño y aplicación de acciones que permitan un mejor desempeño laboral dentro de los diferentes ambientes de aprendizaje, siendo importante a juicio de lanni Gómez (2017), que el docente asuma su rol de asesor investigador para propiciar la transformación educativa.

Así pues se tiene a Marrufo e Ibarra (2012: 21), quienes hacen alocución a lo descrito, destacando que es importante tener habilidades y actitudes que ayuden en el mejoramiento de la educación, dando un crecimiento a la enseñanza dentro de los diferentes ambientes de aprendizaje;

De esta manera, es importante precisar cuál es la misión educativa especifica del docente, y en este contexto, cuales son los conocimientos, capacidades, habilidades y actitudes que correspondan a esas actividades, ya que la misión del docente debería permitir observar el crecimiento de los estudiantes, contribuyendo desde los espacios estructurados para la enseñanza sistemática, al desarrollo integral de las personas, e incorporando sus dimensiones biológicas, afectivas, cognitivas, morales y sociales 


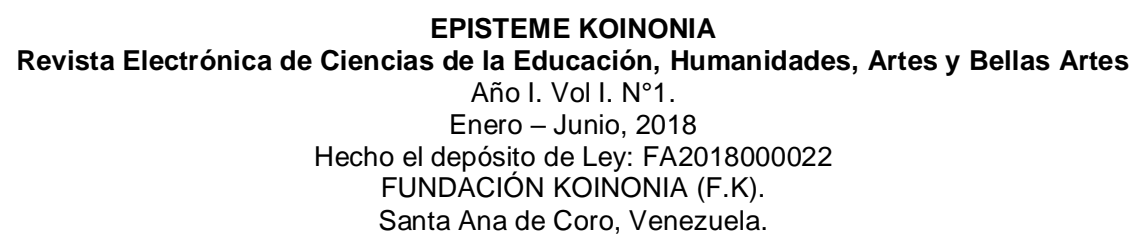

Alvis José Garcia Morales; Caroll Lisette Gonzalez Ten Meer

Destacando esta reflexión dada por el autor, el maestro debe estar a la disposición de servicio educativo, centrado en su rol como educador de saberes bajo un modelo didáctico a seguir por cada estudiante, lo que se convertiría en un aprendizaje significativo contribuyendo de esta manera a una educación de calidad. Al mismo tiempo, este se sería un factor de gran importancia dentro de una educación que cada día reclama docentes con una vocación de servicio creativa, dinámica, con capacidades innatas y propias, que ayuden a descubrir nuevos horizontes, siendo pertinente tener en cuenta Romero \& Villasmil (2017), quienes señalan la necesidad de repensar la formación del docente en la formación universitaria con la finalidad de promover un enfoque transformacional educativo.

\section{CONCLUSIONES}

Uno de los grandes retos es educar a todos y cada uno de los estudiantes con el fin de asegurar que aprendan lo esencial para ejercer los derechos y responsabilidades ciudadanos y para llevar una vida productiva y personalmente satisfactoria. Se han hecho grandes progresos para asegurar la igualdad de oportunidades. Los educadores muestran un creciente interés en el desarrollo y realización de proyectos con herramientas innovadoras que salgan al encuentro de las Diversidad de los alumnos. Así mismo, las adaptaciones a las diversas herramientas mediaran en el desarrollo de habilidades, que permitirán al docente enseñar en clases heterogéneas. La evidencia de la eficacia de estas herramientas y sus adaptaciones, serán muy claras, sin embargo se ha hecho difícil por la falta de un apoyo sistemático al profesorado en esta tarea. Siendo la clave para una realización eficaz los recursos, estrategias o herramientas, además de la preparación de las personas que tienen que llevarlo a la práctica.

Las diferencias individuales en el aprendizaje y las implicaciones que tiene para una educación eficaz han sido siempre objeto, tanto para investigaciones, como de programas y proyectos de innovación destinados a mejorar los resultados escolares. A pesar de los avances en teorías y en investigaciones sobre las diferencias individuales 


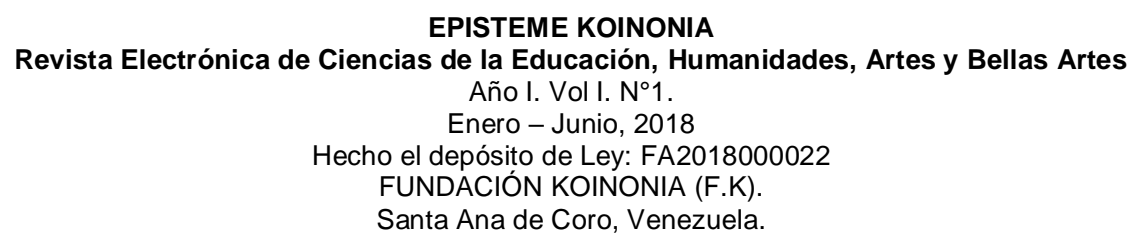

Alvis José Garcia Morales; Caroll Lisette Gonzalez Ten Meer

en el aprendizaje y enseñanza, este conocimiento ha tenido poco efecto en los centros Educativos regulares, existiendo serios problemas para caracterizar la diversidad del alumnado, en muchos casos proyectos y herramientas pedagógicas diseñados para responder han sido contraproducentes, siendo en la práctica poco funcionable. Ahora bien, crear ambientes de aprendizajes y herramientas pedagógicas que correspondan en la práctica a las diversas necesidades de los alumnos y alumnas, ha sido un reto continuo en los esfuerzos de las reformas educativas, y La educación se ha adaptado a las características del alumnado es para el docente una alternativa prometedora para responder a las distintas formas de aprender.

\section{Recomendaciones}

Aprovechar la heterogeneidad de los niños, niñas y adolescentes, para promover aprendizajes cooperativos grupales, que valoren y promuevan su capacidad para apoyarse mutuamente.

Desarrollar actividades de autorregulación del aprendizaje por los propios alumnos, de manera individual o colectiva.

Implementar medidas en el marco de los objetivos curriculares y de su grupo de padres. Las adaptaciones curriculares van de las menos significativas a las más significativas, dependiendo de las necesidades particulares de los niños, niñas y adolescentes.

\section{REFERENCIAS CONSULTADAS}

1. Alfiz, I. (2006). El Proyecto Educativo Escolar. Primera edición. Ediciones Larousse. México. D. F

2. González, Y. (2013). Abordaje de la metodología cualitativa y la investigación-acción para la transformación social. Segunda edición. Ediciones Dabosan, C.A. Caracas. Venezuela.

3. Dos Santos J y Sánchez, S. (2001). Investigación educativa. Cantidad Calidad. Un debate paradigmático. Segunda edición. Ediciones mesa redonda. Magisterio. Bogotá. Colombia. 


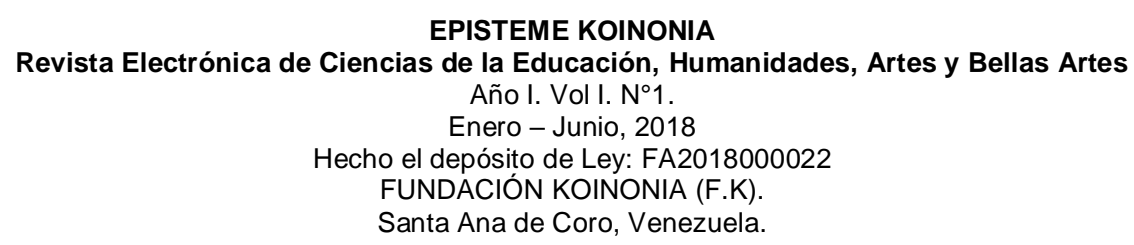

Alvis José Garcia Morales; Caroll Lisette Gonzalez Ten Meer

4. Genesi, M y Suarez, F. (2009). Gestión de calidad del talento humano en las organizaciones educativas inteligentes. Universidad Dr. Rafael Belloso Chacín. www.revistaorbis.org.ve 17 (6) 116-155.

5. Hurtado, J. (2012). El proyecto de investigación. Comprensión holística de la metodología y la investigación. Séptima edición. Ediciones Quiron y Sypal. Caracas. Venezuela.

6. Ianni Gómez., L. (2017). Miramda: Una propuesta educativa emergente desde la investigación. Revista Arbitrada Interdisciplinaria Koinonía, 2(3), 9-30.

Recuperado de http://fundacionkoinonia.com.ve/ojs/index.php/revistakoinonia/article/view/49/36

7. Marrufo, M e Ibarra, Y. (2012). Estrategias didácticas utilizadas para la formación de Estudiantes en lic en educación de la misión sucre. 2011-2012. Trabajo de ascenso publicado. Universidad de Oriente. Cumana.

8. McKernan, J. Investigación-acción y Curriculum. Segunda edición. Ediciones Morata. Madrid. España.

9. Rojas, B. (2010). Investigación cualitativa, fundamentos y praxis. Segunda edición. Ediciones Fondo Editorial de la Universidad Pedagógica Experimental Libertador FEDUPEL. Caracas. Venezuela.

10.Romero, M., \& Villasmil, J. (2017). Repensar la formación docente. Hacia el encuentro de una nueva perspectiva epistémica para su abordaje y resignificación. CIENCIAMATRIA, $3(5), \quad 133-149$. https://doi.org/10.35381/cm.v3i5.17

\section{REFERENCES CONSULTED}

1. Alfiz, I. (2006). The School Educational Project. First edition. Larousse editions. Mexico. D. F

2. González, Y. (2013). Approach to qualitative methodology and action research for social transformation. Second edition. Ediciones Dabosan, C.A. Caracas. Venezuela.

3. Dos Santos J y Sánchez, S. (2001). Educational investigation. Quantity quality. A paradigmatic debate. Second edition. Round table editions. Teaching Bogota Colombia. 


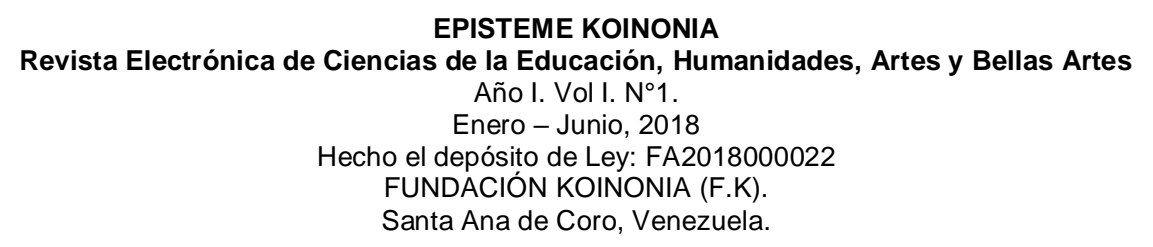

Alvis José Garcia Morales; Caroll Lisette Gonzalez Ten Meer

4. Genesi, M and Suarez, F. (2009). Quality management of human talent in intelligent educational organizations. Dr. Rafael Belloso Chacín University. www.revistaorbis.org.ve 17 (6) 116-155.

5. Marrufo, M and Ibarra, Y. (2012). Didactic strategies used for the formation of Students in lic in education of the mission sucre. 2011-2012. Published promotion work. Eastern University. Cumana

6. McKernan, J. Action Research and Curriculum. Second edition. Morata editions. Madrid. Spain.

7. Rojas, B. (2010). Qualitative research, fundamentals and praxis. Second edition. Editions Editorial Fund of the Libertador Experimental Pedagogical University FEDUPEL. Caracas. Venezuela.

8. Hurtado, J. (2012). The research project Holistic understanding of methodology and research. Seventh edition. Quiron and Sypal editions. Caracas. Venezuela.

(C)2018 por los autores. Este artículo es de acceso abierto y distribuido según los términos y condiciones de la licencia Creative Commons Atribución-NoComercial-Compartirlgual 4.0 Internacional (CC BY-NC-SA 4.0)

(https://creativecommons.org/licenses/by-nc-sa/4.0/). 